\title{
Quality of Learning under an All-Inclusive Approach
}

\author{
José Neves ${ }^{1, *}$, Margarida Figueiredo ${ }^{2}$, Lídia Vicente ${ }^{3}$, Guida Gomes ${ }^{1}$, \\ Joaquim Macedo ${ }^{1}$, and Henrique Vicente ${ }^{4}$ \\ ${ }^{1}$ Algoritmi, Universidade do Minho, Braga, Portugal \\ \{jneves, macedo\}@di.uminho.pt, mguida.mgomes@gmail.com \\ ${ }^{2}$ Departamento de Química, Centro de Investigação em Educação e Psicologia, \\ Escola de Ciências e Tecnologia, Universidade de Évora, Évora, Portugal \\ mtf@uevora.pt \\ ${ }^{3}$ Agrupamento de Escolas de Reguengos de Monsaraz, Reguengos de Monsaraz, Portugal \\ Imrcvicente@gmail.com \\ ${ }^{4}$ Departamento de Química, Centro de Química de Évora, Escola de Ciências e Tecnologia, \\ Universidade de Évora, Évora, Portugal \\ hvicente@uevora.pt
}

\begin{abstract}
Learning, knowledge, educations are syntax forms that stand for a multifaceted matter, and its assets set the advances on culture, organization, and social matters of any society. However, it is not enough just to instruct, it is necessary to do it with quality, in a holistic way, in order to develop academic and social skills. From this point of view, the weight of the formal, non-formal and informal learning contexts should be underlined, and the use of defective information must be emphasizing. Under this setting the assessment to quality of learning is mandatory, although it is hard to do with traditional methodologies for problem solving. Indeed, in this work we will focus on the development of a decision support system, in terms of its knowledge representation and reasoning procedures, under a formal framework based on Logic Programming, complemented with an approach to computing centered on Artificial Neural Networks, to evaluate the Quality of Learning and the respective Degree-ofConfidence that one has on such a happening.
\end{abstract}

Keywords: Learning, Knowledge, Education, Logic Programming, Knowledge Representation and Reasoning, Artificial Neuronal Networks.

\footnotetext{
* Corresponding author.
} 